\title{
Die Digitalisierungsstrategie der Europäischen Union - Meilensteine und Handlungsfelder zwischen digitaler Souveränität und grüner Transformation
}

\author{
Wulf Reiners ${ }^{*}$
}

\begin{abstract}
Digital transformation has been accelerated by the COVID-19 pandemic. Today, it affects almost all areas of social and economic life. As a cross-cutting issue and solution to specific challenges, it is also increasingly the subject of initiatives at EU level. Since 2015, the EU has developed a comprehensive digital agenda that involves various areas, ranging from the single market to foreign and security policy. The paper traces the dynamic development on the basis of strategy documents and policy guidelines in three phases with a focus on 2020 and 2021. It takes stock of the EU's overarching strategy towards digitalisation by examining what the EU understands by it, what its goals are, and what role it draws for itself in shaping the digital transformation. The study shows that the EU tries to grasp digitalisation in a substantial number of policy-specific strategies and guidelines, using mainly four patterns of interpretation - partly in parallel - which differ in terms of geopolitical, environmental, socio-political and economic policy framing.
\end{abstract}

Die COVID-19-Pandemie hat weltweit zu einem einzigartigen Digitalisierungsschub geführt: Privat- und Arbeitsleben wurden in großen Teilen in den virtuellen Raum verlagert. Auch das politische Leben auf EU-Ebene, von Ratspräsidentschaften bis zu Parlamentsdebatten, wurde während der Hochphasen der Pandemie erstmalig überwiegend digital umgesetzt. Die Pandemie bewies, dass digitale Lösungen in Bereichen funktionieren, in denen dies bislang für unmöglich gehalten wurde. Neben der starken Dynamik in den Bereichen Online-Handel (E-Commerce), mobiles Arbeiten und Online-Bildung wurde die Abhängigkeit von Informations- und Kommunikationstechnologien (IKT) sichtbar. In Schulen und öffentlicher Verwaltung zeigte sich großes unerschlossenes Potenzial der Digitalisierung. Hierbei machte der internationale Vergleich erhebliche Defizite auch in vielen europäischen Staaten sichtbar.

Digitalisierung ist ein für zahlreiche miteinander verbundene Phänomene genutzter, umfassender Sammelbegriff, der „die Entwicklung und Anwendung digitaler sowie digitalisierter Techniken, die sich mit allen anderen zivilisatorischen Techniken und Methoden verzahnt und diese erweitert", ${ }^{1}$ umfasst. Die Digitalisierung gibt es demnach nicht, vielmehr entfaltet digitaler technologischer Fortschritt auf vielfältige Weise transformative Wirkung in Gesellschaft, Wirtschaft und Umwelt - von veränderten Kommunikationspraktiken über neuartige industrielle Fertigungsprozesse und Business-Modelle bis hin zu „smarter“ Energienutzung oder Mobilität. Entsprechend werden mit Digitalpolitik sowohl

* Dr. Wulf Reiners, Leitung des „Managing Global Governance“ (MGG) Programms, Deutsches Institut für Entwicklungspolitik, Bonn.

Für die wertvolle Zuarbeit danke ich Lukas Engels und Matthias Kachelmann. Die Studie ist im Rahmen des EU Horizon2020-Projekts „PRODIGEES - Promoting Research on Digitalisation in Emerging Powers and Europe Towards Sustainable Development“ (873119-PRODIGEES-H2020-MSCA-RISE-2019) entstanden.

1 Wissenschaftlicher Beirat der Bundesregierung Globale Umweltveränderungen: Unsere gemeinsame digitale Zukunft, Berlin 2019, S. 1. 
technologische Grundbausteine (etwa digitale Infrastruktur, digitale Services, BreitbandNetzwerke) als auch Bereiche angesprochen, die sich durch Digitalisierung im Wandel befinden. ${ }^{2}$ Wenngleich digitale Instrumente mit der COVID-19-Pandemie an ungeahnter Relevanz gewannen, ist Digitalisierung kein neues Phänomen. Investitionen in Entwicklungen in den Bereichen mobiles Internet, Big Data, künstliche Intelligenz (KI), maschinelles Lernen und Predictive Analytics, Automatisierung und Robotisierung, soziale Medien, Quantentechnologie, Cloud-Computing, 3D-Druck, virtuelle Realität oder Blockchain lösen bereits seit Beginn des 21. Jahrhunderts große Umwälzungen aus.

Damit einhergehend ist Digitalisierung im vergangenen Jahrzehnt weltweit immer umfassender Gegenstand politischer Prozesse geworden, etwa im Zusammenhang mit internationalen Überwachungs- und Spionageskandalen, der Rolle sozialer Medien bei Wahlen und Referenden, dem Schutz personenbezogener Daten im Internet oder multilateralen Verhandlungen um Digitalsteuern. Die Mitgliedstaaten und Institutionen der EU werden sich der Relevanz dieser fundamentalen weltweiten Veränderungen zunehmend bewusst und suchen nach Wegen, die digitale Zukunft Europas und relevante globale Prozesse zu gestalten. Die Herausforderungen für die EU sind dabei so groß wie die Chancen, die eine erfolgreiche digitale Transformation versprechen.

Auf der einen Seite stehen die Verheißungen und Potenziale, die digitaler Wandel verspricht. Digitalisierung kann dringend nötige Emissionsreduktionen in der europäischen Wirtschaft durch eine schnellere Dekarbonisierung erzielen und die beabsichtige Etablierung von Kreislaufwirtschaft und umweltschonender Landwirtschaft unterstützen. Digitale Innovationen können Wissen weltweit zugänglich, öffentliche Verwaltungen transparenter und effizienter und weitreichende politische Partizipation möglich machen. Sie vermögen inter- und transnationale Kooperation und das Zusammengehörigkeitsgefühl, in Europa und weltweit, durch die Entwicklung einer „vernetzte[n] Gesellschaft“3 zu stärken. Wirtschaftlich verspricht eine deutliche Steigerung der digitalen Performance und Wettbewerbsfähigkeit einen Pro-Kopf-Anstieg des Bruttoinlandsprodukt (BIP) in der EU um 7,2 Prozent bis zum Jahr $2027 .{ }^{4}$

Gleichzeitig birgt der digitale Wandel erhebliche Risiken kurz- und langfristiger Natur. Der bereits heute enorme und weiter ansteigende Energieverbrauch von IKT, der auch aus nicht-nachhaltigen Quellen gedeckt wird, die wachsende soziale Ungleichheit in vielen Ländern oder der drohende massenhafte Wegfall von Arbeitsplätzen durch Automatisierung gehören dazu. Der europäischen Wirtschaft schaden Cyberattacken auf Informations- und Produktionssysteme in Form von Diebstahl, Spionage und Sabotage. Beispiele wie das Brexit-Referendum oder die COVID-19-Impfungen zeigen, wie demokratische Prozesse und gesellschaftliche Diskurse durch soziale Medien und Desinformationskampagnen beeinflusst werden.

Im internationalen Vergleich droht die EU den Anschluss an den technologischen Fortschritt zu verlieren, ${ }^{5}$ und damit Gestaltungsmacht für wegweisende Entwicklungen des 21 .

2 Falk Steiner/Viktoria Grzymek: Digital Sovereignty in the EU. Vision Europe, Gütersloh 2020, S. 2.

3 Wissenschaftlicher Beirat der Bundesregierung Globale Umweltveränderungen: Unsere gemeinsame digitale Zukunft, 2019, S. 9.

4 Deloitte LLP (Hrsg.): Digitalisation. An opportunity for Europe, London 2021, S. 5.

5 Europäische Kommission: I-DESI 2020: How digital is Europe compared to other major world economies?, 17. Dezember 2020, LC - 01432083. 
Jahrhunderts. Bahnbrechende digitale Innovationen werden insbesondere in den USA und China entwickelt und die Investitionen europäischer Firmen in Schlüsseltechnologien wie Quantentechnologie oder KI sind nur ein Bruchteil im Vergleich zu US-amerikanischen und chinesischen Beiträgen. ${ }^{6}$ Die meisten EU-Staaten gehören im internationalen Vergleich zur Gruppe der fortgeschritten digitalisierten Länder, doch wandelt sich das digitale Umfeld hier erheblich langsamer als in aufstrebenden Mächten wie China, Indonesien oder Indien. ${ }^{7}$

Auf EU-Ebene lassen sich seit Mitte der 2010er Jahre verstärkt Initiativen im Bereich Digitalisierung und Digitalwirtschaft beobachten, um Rahmenbedingungen zu schaffen, die positiven Potenziale der Digitalisierung zu heben und negative Folgen zu verhindern bzw. abzumildern. Unter der aktuellen Präsidentin Ursula von der Leyen hat sich die Europäische Kommission für die aktuelle Legislaturperiode (2019-2024) die Priorität gesetzt, ein Europa „fit für das digitale Zeitalter“ zu schaffen, das es den BürgerInnen, der Wirtschaft und den Regierungen ermöglichen soll, umfassend von den Instrumenten und Möglichkeiten der digitalen Welt zu profitieren. Das kommende Jahrzehnt wurde zur „Digitalen Dekade Europas" erklärt und ein Bündel von Initiativen in einer Vielzahl von Themen und Zielsetzungen angestoßen. ${ }^{8}$ Digitalisierung wird dabei sowohl als eigener Politikbereich skizziert als auch querschnittsmäßig in Strategien enger definierter Politikfelder aufgegriffen.

Die Koordination von Politiken und Ressourcenallokation auf Grundlage politischer Leitlinien und Zielsetzungen, die wiederum Antwort auf eine Problemanalyse darstellen, gehören zu den Kernelementen politischer Strategien. ${ }^{9}$ Ihre Analyse gibt Aufschluss darüber, was die EU unter Digitalisierung versteht und welche Rolle sie sich bei ihrer Gestaltung zuschreibt. In diesem Sinne geht der Beitrag in drei Analyseschritten einer übergreifenden europäischen Digitalisierungsstrategie auf den Grund, auch wenn die EU das Feld in politikfeldspezifischen Initiativen und Einzelstrategien bearbeitet. Dafür wird in einem ersten Schritt dargestellt, wie sich die digitale Agenda auf EU-Ebene seit 2015 entwickelt hat. Auf dieser Grundlage nimmt der Beitrag eine Kategorisierung des heutigen Aktivitätsspektrums vor, d. h. derjenigen Teilbereiche der Digitalisierung in einem potenziell weiten Feld, in dem die EU schwerpunktmäßig aktiv geworden ist oder werden will. In einem abschließenden Schritt werden vier idealtypische Lesarten der Digitalisierung und der Rolle der EU herausgearbeitet, die sich entlang ihres politischen Framings also entlang der Betonung bestimmter Aspekte zugunsten eines bestimmten Interpretationsschemas - unterscheiden lassen. ${ }^{10}$

6 Europäische Kommission: Rethinking Strategic Autonomy in the Digital Age, European Political Strategic Centre: EPSC Strategic Notes, Juli 2019.

7 Bhaskar Chakravorti/Ravi Shankar Chaturvedi/Christina Filipovic/Griffin Brewer: Digital in the Age of Covid. Trust in the Digital Economy and Its Evolution Across 90 Economies as the Planet Paused for a Pandemic, Medford 2020, S. 24.

8 Europäische Kommission: Ein Europa für das digitale Zeitalter - Eine neue Generation von Technologien für die Menschen, 2021, abrufbar unter: https:/ec.europa.eu/info/strategy/priorities-2019-2024/europe-fit-digital -age_de (letzter Zugriff: 18.10.2021).

9 Richard Rumelt: Good strategy/Bad strategy. The difference and why it matters, London 2011.

10 Zum politischen Framing vgl. Robert M. Entman: Framing: Toward Clarification of a Fractured Paradigm, in: Journal of Communication 4/1993, S. 51-58; Merlijn Van Hulst/Dvora Yanow: From Policy "Frames" to "Framing": Theorizing a More Dynamic, Political Approach, in: The American Review of Public Administration $1 / 2014$, S. $92-112$. 
Vor dem Hintergrund, dass politische Strategien den Zweck verfolgen, die zentralen Problemstellungen eines Politikfeldes zu erfassen und einen Weg für Maßnahmen vorzuzeichnen, die die Herausforderungen in koordinierter und fokussierter Weise adressieren sollen, legt die Untersuchung besonderes Augenmerk auf drei konzeptionelle Kernelemente: (1) Die Situationsanalyse erfasst, wie die EU die Ausgangslage wahrnimmt und vor welche Probleme und Chancen sie sich durch die Digitalisierung gestellt sieht. (2) Die Analyse der Zielformulierung geht der Frage nach, was die EU sich vornimmt, um der Situationsanalyse entsprechend zu begegnen. (3) Mit Blick auf Zielimage und Zielgruppenbotschaft wird schließlich untersucht, welches Selbstbild die EU gegenüber Gesellschaft und Dritten skizziert. ${ }^{11}$ Die Analyse erfolgt auf Grundlage von EU-Dokumenten übergreifender Natur zwischen 2015 und 2021; insbesondere Strategiepapiere, Rahmenprogramme und Schlussfolgerungen des Europäischen Rats. Sie werden verstanden als die maßgeblichen politischen Wegweiser, entlang derer sich das heutige Handlungsspektrum entfaltet hat.

\section{Phasen und Meilensteine der europäischen Digitalagenda}

Die EU verfügt heute über eine umfassende Digitalagenda, die sich über zahlreiche Politikfelder erstreckt. Anhand politischer Leitlinien und zentraler Initiativen lassen sich Phasen der Entwicklung und Meilensteine identifizieren, die für ihre heutige Ausgestaltung wegweisend waren. Sie bieten Einsichten in die Prioritäten und die Begründungszusammenhänge bei der Entwicklung der Agenda in den vergangenen Jahren. Die wegweisenden Rahmenwerke lassen sich grob in drei - sich zum Teil leicht überschneidende - Phasen unterteilen.

\section{5 bis 2017: digitaler Binnenmarkt}

Ein Schlüsselwerk der ersten Entwicklungsphase (2015-2017) ist die Strategie für den digitalen Binnenmarkt der Europäischen Kommission aus dem Jahr 2015. Das Dokument ging von der zentralen Situationsanalyse der Staats- und Regierungschefs aus, dass „[d]igitale Technologien [...] immense Chancen für Innovation, Wachstum und Arbeitsplätze"12 böten, die Aussicht auf eine Expansion von Märkten und bessere Dienstleistungen zu günstigeren Preisen bereithalten könnten. Mit dem Ziel, diese wirtschaftlichen Potenziale nutzbar zu machen, sollte die Förderung der vier Grundfreiheiten des Binnenmarkts, ein günstiges Investitionsklima für digitale Netze, Forschung und Unternehmen und europäische Verbraucher- und Datenschutzstandards miteinander verbunden werden. Die Strategie definierte in einer umfassenden Ausrichtung diverse Handlungsfelder vom Online-Handel über Datenschutz bis hin zu digitalen Kompetenzen und elektronischen Behördendiensten (e-Government). Sie war in den folgenden Jahren Orientierungspunkt für zahlreiche konkrete Initiativen, darunter 30 Legislativvorschläge. Dazu gehörten die Datenschutzgrund-

11 Peter Schröder: Politische Strategien, Potsdam 2011, S. 51-64.

12 Europäischer Rat: Tagung des Europäischen Rates (25. und 26. Juni 2015) - Schlussfolgerungen, EUCO 22/15, S. 6. 
verordnung (DSGVO), ${ }^{13}$ Maßnahmen zur Förderung von E-Commerce ${ }^{14}$ und des Aufbaus einer europäischen Datenwirtschaft, ${ }^{15}$ ein Aktionsplan für digitale Bildung ${ }^{16}$ sowie Maßnahmen in den Bereichen Konnektivität, Breitbandversorgung und fünfte Generation des Mobilfunks $(5 \mathrm{G})^{17}$ sowie Cybersicherheit. ${ }^{18}$

Im Jahr 2017 erreichte der Schwerpunkt „Digitales Europa“ bis dahin ungekannte Präsenz im Rahmen der Ratspräsidentschaft Estlands, das als „weitest fortgeschrittene digitale Gesellschaft der Welt "19 eine führende Rolle in Digitalisierungsfragen, insbesondere behördlichen Dienstleistungen, einnahm. Auf dem Tallinner Digitalgipfel identifizierten die europäischen Staats- und Regierungschefs umfassende Dringlichkeiten. Dazu gehörten die Regulierung von Online-Plattformen und die Anpassung des Steuersystems, die Bekämpfung von Terrorismus im Internet sowie "neue Trends“ wie KI oder Blockchain. ${ }^{20}$ Über die Debatte um die Zukunft Europas und die Halbzeitüberprüfung der Strategie für den digitalen Binnenmarkt hinaus wurden zuvor etablierte Prioritäten wie Datenwirtschaft, Förderung von Digitalunternehmen und Wettbewerbsfähigkeit, Investitionen in digitale Infrastruktur und Forschung fortgeschrieben, ergänzt um Überlegungen zu den Auswirkungen der Digitalisierung auf Beschäftigung, lebenslanges Lernen und neue soziale Rechte.

\section{7 bis 2020: außen- und sicherheitspolitische Herausforderungen}

Standen von 2015 bis ins Jahr 2018 hinein die EU-internen gesellschaftlichen und wirtschaftlichen Entwicklungen als digitaler Binnenmarkt im Zentrum der politischen Leitlinien, setzte am Ende dieser Phase parallel eine Erweiterung und neue Betonung der Perspektive auf die außen- und sicherheitspolitische Dimension von Digitalisierung ein. Dazu gehörten nicht nur der Datenverkehr mit Drittstaaten in Handelsabkommen, sondern auch Fragen zu Cybersicherheit, hybriden Bedrohungen und Spionageabwehr.

13 Europäisches Parlament/Rat der Europäischen Union: Verordnung (EU) 2016/679 des Europäischen Parlaments und des Rates vom 27. April 2016 zum Schutz natürlicher Personen bei der Verarbeitung personenbezogener Daten, zum freien Datenverkehr und zur Aufhebung der Richtlinie 95/46/EG (Datenschutz-Grundverordnung), in: Amtsblatt der EU, Nr. L 119/1 vom 4. Mai 2016, 2016/679.

14 Europäische Kommission: Mitteilung der Kommission an das Europäische Parlament, den Rat, den Europäischen Wirtschafts- und Sozialausschuss und den Ausschuss der Regionen. Ein umfassendes Konzept zur Förderung des grenzüberschreitenden elektronischen Handels für die Bürger und Unternehmen Europas, 25. Mai 2016, COM(2016) 320 final.

15 Europäische Kommission: Mitteilung der Kommission an das Europäische Parlament, den Rat, den Europäischen Wirtschafts- und Sozialausschuss und den Ausschuss der Regionen. - „Aufbau einer Europäischen Datenwirtschaft", 10. Januar 2017, COM(2017) 9 final.

16 Europäische Kommission: Mitteilung der Kommission an das Europäische Parlament, den Rat, den Europäischen Wirtschafts- und Sozialausschuss und den Ausschuss der Regionen zum Aktionsplan für digitale Bildung, 17. Januar 2018, $\operatorname{COM}(2018) 22$ final.

17 Europäische Kommission: Mitteilung der Kommission an das Europäische Parlament, den Rat, den Europäischen Wirtschafts- und Sozialausschuss und den Ausschuss der Regionen. Konnektivität für einen wettbewerbsfähigen digitalen Binnenmarkt - Hin zu einer europäischen Gigabit-Gesellschaft, 15. September 2016, $\operatorname{COM}(2016) 587$ final.

18 Europäische Kommission/Hohe Vertreterin der Europäischen Union für Außen- und Sicherheitspolitik: Gemeinsame Mitteilung an das Europäische Parlament, den Rat, den Europäischen Wirtschafts- und Sozialausschuss und den Ausschuss der Regionen. Abwehrfähigkeit, Abschreckung und Abwehr: die Cybersicherheit in der EU wirksam erhöhen, 13. September 2017, JOIN(2017) 450 final.

19 Matt Reynolds: Welcome to E-stonia, the world's most digitally advanced society, in: Wired, 20. Oktober 2016.

20 Europäischer Rat: Tagung des Europäischen Rates (19. Oktober 2017) - Schlussfolgerungen, EUCO 14/17. 
Bereits 2013 war mit der EU-Cybersicherheitsstrategie die stärkere Ausrichtung der politischen Leitlinien auf sicherheitspolitische Implikationen deutlich geworden. ${ }^{21} \mathrm{Im}$ selben Jahr waren auch die Umwandlung der Europäischen Agentur für Netz- und Informationssicherheit zur Europäischen Agentur für Cybersicherheit (ENISA) beschlossen und ein Vorschlag für den ersten Rechtsakt der Union zur Cybersicherheit, die Richtlinie über die Sicherheit von Netz- und Informationssystemen, erarbeitet worden. ${ }^{22}$ Auf dieser Grundlage und im Einklang mit den Digitalisierungsbezügen in der Globalen Strategie der EU erfuhr die Thematik ab 2017 eine neue Dynamik. Mit dem Verständnis, dass Cybersicherheit u. a. ein Schlüssel für „den Wohlstand, das Wachstum und die Sicherheit in der EU und für die Integrität unserer freien und demokratischen Gesellschaften “23 sei, verständigte die EU sich auf umfangreiche Maßnahmen im Bereich Cybersicherheitskapazitäten - einschließlich der weiteren Stärkung der ENISA - und entwickelte Pläne für eine koordinierte Reaktion auf große Cybersicherheitsvorfälle und -krisen. ${ }^{24}$ Diese umfassten eine Aktualisierung des EU-Politikrahmens für die Cyberabwehr ${ }^{25}$ und mündeten in eine neue Cybersicherheitsstrategie für die digitale Dekade, um auf die technische Entwicklung und veränderte Bedrohungslage zu reagieren. Die Strategie verbindet Netz- und Informationssicherheit sehr umfassend mit „Verkehr, Energie und Gesundheit, Telekommunikation, Finanzen, Sicherheit, demokratische[n] Prozesse[n], Raumfahrt und Verteidigung "26 und adressiert u. a. den Schutz zentraler öffentlicher und privater Infrastruktur (z. B. 5G, Krankenhäuser, Energieversorgung, Schienennetz).

Digitalisierung wurde so verstärkt in den Zusammenhang mit den Plänen für eine europäische Sicherheitsunion gestellt. Gleichzeitig erweiterten sich die Bezüge zur Position der EU in der Welt. Mit der "Cyber Diplomacy Toolbox“ installierten die Mitgliedstaaten ein neues Instrumentarium, das rasche und gemeinsame diplomatische Antworten der

21 Europäische Kommission/Hohe Vertreterin der Europäischen Union für Außen- und Sicherheitspolitik: Gemeinsame Mitteilung an das Europäische Parlament, den Rat, den Europäischen Wirtschafts- und Sozialausschuss und den Ausschuss der Regionen. Cybersicherheitsstrategie der Europäischen Union - ein offener, sicherer und geschützter Cyberraum, 7. Februar 2013, JOIN(2013) 1 final.

22 Europäisches Parlament/Rat der Europäischen Union: Richtlinie (EU) 2016/1148 des Europäischen Parlaments und des Rates vom 6. Juli 2016 über Maßnahmen zur Gewährleistung eines hohen gemeinsamen Sicherheitsniveaus von Netz- und Informationssystemen in der Union, in: Amtsblatt der EU, Nr. L 194/1 vom 19. Juli 2016.

23 Rat der Europäischen Union: Schlussfolgerungen des Rates zur Gemeinsamen Mitteilung an das Europäische Parlament und den Rat. „Abwehrfähigkeit, Abschreckung und Abwehr: die Cybersicherheit in der EU wirksam erhöhen“ - Schlussfolgerungen des Rates (20. November 2017), 14435/17.

24 Europäische Kommission/Hohe Vertreterin der Europäischen Union für Außen- und Sicherheitspolitik: Abwehrfähigkeit, Abschreckung und Abwehr: die Cybersicherheit in der EU wirksam erhöhen, September 2017; Europäisches Parlament/Rat der Europäischen Union: Verordnung (EU) 2019/881 des Europäischen Parlaments und des Rates vom 17. April 2019 über die ENISA (Agentur der Europäischen Union für Cybersicherheit) und über die Zertifizierung der Cybersicherheit von Informations- und Kommunikationstechnik und zur Aufhebung der Verordnung (EU) Nr. 526/2013 (Rechtsakt zur Cybersicherheit), in: Amtsblatt der EU, Nr. L 151/15 vom 7. Juni 2019, 2019/881.

25 Rat der Europäischen Union: EU-Politikrahmen für die Cyberabwehr, 18. November 2014, 15585/14; Rat der Europäischen Union: EU-Politikrahmen für die Cyberabwehr (Aktualisierung 2018), 19. November 2018, $14413 / 18$.

26 Europäische Kommission/Hoher Vertreter der Europäischen Union für Außen- und Sicherheitspolitik: Gemeinsame Mitteilung an das Europäische Parlament, den Rat, den Europäischen Wirtschafts- und Sozialausschuss und den Ausschuss der Regionen. Die Cybersicherheitsstrategie der EU für die digitale Dekade, 16. Dezember 2020, JOIN(2020) 18 final, S. 1. Für eine Analyse der Strategie vgl. auch Annegret Bendiek/ Matthias C. Kettemann: Revisiting the EU Cybersecurity Strategy: A Call for EU Cyber Diplomacy, Stiftung Wissenschaft und Politik: SWP Comments 16/2021, S. 1-8. 
EU auf böswillige Cyberangriffe ermöglichen soll, gegebenenfalls auch unter Nutzung der Gemeinsamen Außen- und Sicherheitspolitik (GASP). ${ }^{27}$ Mit ihrem Ansatz zur digitalen Diplomatie drückte die EU Führungsambitionen aus zugunsten einer „offenen und transparenten, regelbasierten und sicheren, vertrauenswürdigen und menschenzentrierten sowie für alle zugänglichen globalen digitalen Wirtschaft" ${ }^{\text {"28 }}$ und bei der Gestaltung globaler Regeln und Standards in internationalen Organisationen und multilateralen Foren. ${ }^{29}$

Neben Cybersicherheitskapazitäten und Cyberdiplomatie erlangte strategische Kommunikation an neuer Bedeutung in der Problemanalyse - nicht zuletzt aufgrund der russischen Desinformationskampagnen im Zusammenhang mit der Ukraine-Krise (seit 2014). Vor diesem Hintergrund baute die EU u. a. die „East StratCom Task Force“ und weitere geographisch spezialisierte Arbeitsgruppen für strategische Kommunikation im Europäischen Auswärtigen Dienst (EAD) auf und entwickelte einen Aktionsplan gegen Desinformation. ${ }^{30}$ Die Maßnahmen wurden auch mit Blick auf die Europawahl 2019 entwickelt, um falschen oder irreführenden Informationen zu begegnen, welche durch die gezielte Nutzung sozialer Medien über das Potenzial verfügen, öffentlichen Schaden anzurichten. Desinformationskampagnen können zudem Teil hybrider Kriegsführung sein. ${ }^{31}$

\section{Seit 2020: digitale Dekade, Green Deal und COVID-19-Krise}

Mit Beginn der aktuellen Legislaturperiode (2019-2024) gewann Digitalisierung an zusätzlicher Bedeutung auf EU-Ebene. Mit seiner „Neuen Strategischen Agenda 20192024“ führte der Europäische Rat zwar im Wesentlichen die Betonung bestehender Handlungsfelder fort. Gleichzeitig verankerten die Staats- und Regierungschefs in ihrem neuen Gesamtrahmen jedoch prominent die Forderung nach „digitale[r] Souveränität“ und der „Verwirklichung eines klimaneutralen, grünen, fairen und sozialen Europas“32 An diesen beiden Forderungen orientieren sich seitdem die Digitalagenda und die politische Rhetorik in der EU.

In ihren politischen Leitlinien für die künftige Kommission formulierte die neue Präsidentin ein „Europa, das für das digitale Zeitalter gerüstet ist“33 als eines ihrer sechs übergeordneten Ziele. Europas Souveränität in kritischen Technologiebereichen durch Investitionen und das gemeinsame Setzen von Standards mit internationaler Vorbildwirkung, u. a. im Bereich der KI, finden sich hier ebenso wieder wie weitere Schritte zur Vollendung des digitalen Binnenmarktes, der Schutz vor Cyberbedrohungen und die digitale Aus- und Fortbildung. Digitalisierung wird hier als parallele Gestaltungspriorität zum europäischen

27 Rat der Europäischen Union: Entwurf von Schlussfolgerungen des Rates über einen Rahmen für eine gemeinsame diplomatische Reaktion der EU auf böswillige Cyberaktivitäten ("Cyber Diplomacy Toolbox") - Annahme, 7. Juni 2017, 9916/17.

28 Rat der Europäischen Union: Schlussfolgerungen des Rates zur digitalen Diplomatie, 17. November 2020, $12804 / 20$, S. 3.

29 Ebenda.

30 Europäische Kommission/Hohe Vertreterin der Europäischen Union für Außen- und Sicherheitspolitik: Gemeinsame Mitteilung an das Europäische Parlament, den Rat, den Europäischen Wirtschafts- und Sozialausschuss und den Ausschuss der Regionen. Aktionsplan gegen Desinformation, 5. Dezember 2018, JOIN(2018) 36 final.

31 Ebenda, S. 3; Europäischer Rat: Tagung des Europäischen Rates (20. Juni 2019) - Schlussfolgerungen, EUCO $9 / 19$.

32 Ebenda.

33 Ursula von der Leyen: A Union that strives for more. My agenda for Europe, 16. Juli 2019. 
Grünen Deal (Green Deal) angelegt, der als wirtschaftliche und gesellschaftliche Transformationsstrategie den klima- und umweltbedingten Herausforderungen begegnen soll.

Der Green Deal selbst weist zahlreiche Verweise auf digitale Instrumente im Zusammenhang mit dem Schutz natürlicher Ökosysteme, der nachhaltigen Nutzung von Ressourcen und der Verbesserung der menschlichen Gesundheit auf. Technologie wird als wesentliche Voraussetzung für die angestrebten Veränderungen im Sinne der Klima- und Nachhaltigkeitsziele verstanden - sei es bei der Digitalisierung des Energiemarktes zur Verbesserung von Energieeffizienz, in der neuen Industriestrategie und dem Übergang zu Kreislaufwirtschaft, der Bereitstellung von Verbraucherinformationen zu Lebensmitteln, der Verfügbarkeit von Umweltdaten durch Fernüberwachung, der Versorgung mit Rohstoffen, vernetzte Mobilität sowie Innovation und faktengestützte Entscheidungsfindung auf Grundlage zugänglicher Daten. ${ }^{34}$ Am Green Deal zeigt sich die enorme Relevanz, die der Digitalisierung in praktisch allen zentralen Handlungsfeldern auf europäischer Ebene zugeschrieben wird.

Auf Grundlage dieser politischen Leitlinien entfachte sich auf EU-Ebene in den Jahren 2020 und 2021 ein regelrechtes Feuerwerk an Initiativen. Auftakt war die Vision der Kommission für die „Gestaltung der digitalen Zukunft Europas“. Das fundamentale Konzept umfasst drei Säulen:

(1) digitale Kompetenzen, Schutz vor Cyberbedrohungen, Entwicklung von KI, ultraschnelle Breitbandverbindungen, Ausbau von Hochleistungsrechenkapazitäten als „Technologie im Dienste der Menschen“.

(2) „Eine faire und wettbewerbsfähige Wirtschaft“ durch Förderung von Start-ups und kleiner Unternehmen, Regulierung von Online-Plattformen und des Wettbewerbs im Binnenmarkt; Verbesserung des Zugangs zu hochwertigen Daten.

(3) „Eine offene, demokratische und nachhaltige Gesellschaft“ durch die Verbindung von Initiativen zur Verwirklichung der Klimaneutralität, Nutzung von Gesundheitsdaten, Datenschutz und Bekämpfung von Desinformation. ${ }^{35}$

Zusammen mit einer europäischen Datenstrategie ${ }^{36}$ und einem Weißbuch zur $\mathrm{KI}^{37}$ stellte die Kommission im Februar 2020 dieses Konzept vor. Kurz danach begann die COVID-19-Pandemie das politische Geschehen in Europa zu dominieren. Die Mitgliedstaaten verbanden in der Folge den digitalen Wandel mit der wirtschaftlichen Erholung von den Auswirkungen der Pandemie. ${ }^{38}$ Die Digitalisierung des Binnenmarktes, digitale Souveränität und die Führungsrolle in strategischen internationalen digitalen Wertschöpfungsketten wurden dabei zunehmend an Forschung und Innovation gekoppelt, wobei Grundlagentechnologien (Hochleistungsrechnen, Quantentechnologien und Cloud-Com-

34 Europäische Kommission: Mitteilung der Kommission an das Europäische Parlament, den Rat, den Europäischen Wirtschafts- und Sozialausschuss und den Ausschuss der Regionen. Der europäische Grüne Deal, 11. Dezember 2019, COM(2019) 640 final, S. 4.

35 Europäische Kommission: Mitteilung der Kommission an das Europäische Parlament, den Rat, den Europäischen Wirtschafts- und Sozialausschuss und den Ausschuss der Regionen. Gestaltung der digitalen Zukunft Europas, 19. Februar 2020, $\operatorname{COM}(2020) 67$ final.

36 Europäische Kommission: Mitteilung der Kommission an das Europäische Parlament, den Rat, den Europäischen Wirtschafts- und Sozialausschuss und den Ausschuss der Regionen. Eine europäische Datenstrategie, 19. Februar 2020, $\operatorname{COM(2020)~} 66$ final.

37 Europäische Kommission: Weißbuch. Zur Künstlichen Intelligenz - ein europäisches Konzept für Exzellenz und Vertrauen, 19. Februar 2020, $\operatorname{COM}(2020) 65$ final.

38 Europäischer Rat: Gemeinsame Erklärung der Mitglieder des Europäischen Rates, 26. März 2020, S. 6. 
puting) sowie Breitbandnetze und Netzanbindung auch direkt eine Rolle bei der Bewältigung der Pandemie zugeschrieben wurden - nicht zuletzt mit Blick auf Fernunterricht und Telearbeit. In ihrer umfassenden Agenda bestätigten die Mitgliedstaaten auch weitere zentrale Handlungsfelder (Cybersicherheit, Plattformwirtschaft, öffentliche Dienste, Kompetenzen) und ergänzten diese um neue Bausteine aus den Bereichen Medienpolitik und Besteuerung der digitalen Wirtschaft. Mit einem Abschnitt zu ökologischer Nachhaltigkeit, der auf Beiträge des digitalen Ökosystems zum Ziel einer klimaneutralen EU dringt, schrieb man die Verbindung von Digitalisierung mit der Klima- und Nachhaltigkeitsdebatte fort. ${ }^{39}$

Der Digitalisierungsschwerpunkt der neuen Kommission und die COVID-19-Pandemie führten zu einer Erweiterung der Digitalstrategie und politischem Rückenwind gleichermaßen. Dies schlug sich in den Verhandlungen für den mehrjährigen Finanzrahmen (MFR) und dem Aufbauinstrument „NextGenerationEU“ (NGEU) nieder. Das Paket von 2,018 Billionen Euro weist zahlreiche prominente Digitalisierungsbezüge auf, wobei die zentrale Zielsetzung einer grüneren, digitaleren und widerstandsfähigeren PostCOVID-19-EU deutlich hervorgehoben wird. Digitales soll in allen Programmen Priorität haben und Investitionen in diesem Bereich sollen mindestens 20 Prozent der Aufbau- und Resilienzfazilität, mit 672,5 Milliarden Euro das Herzstück von NGEU, ausmachen. ${ }^{40}$ Auch wird für eine positive Bewertung der nationalen Reform- und Investitionsagenden, die von den Mitgliedstaaten vor der NGEU-Mittelzuweisung vorgelegt werden müssen, ihr Beitrag zur digitalen Wende als Voraussetzung benannt. ${ }^{41}$

Ein genauer Blick in die finanziellen Zusagen 2021 bis 2027 (MFR und NGEU) zeigt, dass der digitale Wandel als eine von fünf Prioritäten festgeschrieben wird, die aus verschiedenen Finanzlinien bedient wird. Im Zentrum steht dabei die Rubrik „Binnenmarkt, Innovation und Digitales“ mit einem MFR-Gesamtvolumen in Höhe von 149,51 Milliarden Euro (zzgl. 11,49 Milliarden Euro aus NGEU). Das Instrument „Europäische strategische Investitionen“ umfasst nicht nur die Fazilität „Connecting Europe Digital“, die gut 2 Milliarden Euro für die EU-weite Einführung von Gigabit-Netzen und den Zugang zu digitalen Infrastrukturen vorsieht. Es weist zudem 7,59 Milliarden Euro für das Programm „Digitales Europa“ aus, die dem Aufbau von Innovationhubs und Kapazitäten in den Bereichen KI, Hochleistungsrechnen, Cybersicherheit und digitale Kompetenzen dienen sollen. ${ }^{42}$ Der InvestEU-Fonds, der über 372 Milliarden Euro an öffentlichen und privaten Investitionen mobilisieren soll, unterstützt im Politikbereich „Forschung, Innovation und Digitalisierung" durch EU-Haushaltsgarantien in Höhe von 6,6 Milliarden Euro (Richtwert) u. a. Projekte zur Digitalisierung der Industrie und KI. ${ }^{43}$ Hinzu kommen nicht weiter spezifizierte Budgetanteile für Maßnahmen im Bereich Digitales insbesondere im „Instrument für technische Unterstützung“ und in den Budgetlinien „Forschung und Innovation“

39 Rat der Europäischen Union: Gestaltung der digitalen Zukunft Europas - Schlussfolgerungen des Rates (9. Juni 2020), 8711/20.

40 Europäische Kommission: The EU's 2021-2027 long-term Budget and NextGenerationEU. Facts and figures, Factsheet, Luxemburg 2021.

41 Europäischer Rat: Außerordentliche Tagung des Europäischen Rates (17., 18., 19., 20. und 21. Juli 2020) Schlussfolgerungen, EUCO 10/20, S. 6.

42 Europäische Kommission: The EU's 2021-2027 long-term Budget and NextGenerationEU. Facts and figures, Factsheet, Luxemburg 2021.

43 Europäische Kommission: Fragen und Antworten: Programm „InvestEU“, 9. März 2021, QANDA/21/1045. 
sowie Kohäsionspolitik. Darüber hinaus sind aufgrund der jeweiligen inhaltlichen Agenden Anteile der Budgets für "Sicherheit und Verteidigung“, „Nachbarschaft und die Welt" und die „Europäische öffentliche Verwaltung“ anzurechnen, was den Querschnittcharakter des digitalen Wandels unterstreicht. Auch auf der Einnahmenseite spielt Digitalisierung mit der Digitalabgabe als neue Einnahmequelle zwecks Rückzahlung der NGEU-Mittel eine wichtige Rolle, um die „Verwirklichung der digitalen Dekade Europas auf ein solides finanzielles Fundament zu stellen“44.

Der Europäische Rat baute die Leitlinien für eine EU-Digitalisierungsstrategie in seinem Sondergipfel Anfang Oktober 2020 weiter aus. In der Forderung nach einem „digitalen Kompass" bestärkte er die Verbindung des Übergangs zu einer grünen Wirtschaft mit dem digitalen Wandel und benannte als Schwerpunkte die Regulierung der systemischen Rolle von Online-Plattformen, das Schmieden neuer Industrieallianzen in digitalen Schlüsseltechnologien, einschließlich der Förderung von Batterie- und Mikroelektronik als Vorhaben von gemeinsamem europäischem Interesse. Vor dem Hintergrund der COVID-19Pandemie wurden die Stärkung der Wettbewerbsfähigkeit, einschließlich der europäischen Datenwirtschaft, und die „Errichtung eines wirklich digitalen Binnenmarkts“ betont und zugleich mit der Zielsetzung der „digitalen Souveränität“ ${ }^{45}$ der EU verbunden, die nur auf Grundlage autonomer technologischer Entscheidungen und strategischer digitaler Kapazitäten und Infrastrukturen zu gewährleisten sei. In den Schlussfolgerungen der weiteren Treffen 2021 hoben die Staats- und Regierungschefs erneut die Außen- und Sicherheitsdimensionen der EU-Digitalisierungsstrategie hervor. So befanden sie für die Beziehungen zu Afrika u. a. die Zusammenarbeit im Bereich der digitalen Wirtschaft für maßgeblich. ${ }^{46}$ Außerdem betonten sie die Notwendigkeit der Bekämpfung illegaler und insbesondere terroristischer Online-Inhalte sowie von Radikalisierung im Internet. ${ }^{47}$

Ein Jahr nach Vorstellung der Digitalstrategie führte die Kommission im Frühjahr 2021 die zahlreichen Anstöße der vorangegangenen Monate in einen Gesamtentwurf über. Zielvorstellungen und Wege für den digitalen Wandel in Europa bis 2030 fasste sie in zwei großen Bereichen zusammen: europäische digitale Grundsätze und digitaler Kompass. ${ }^{48}$ Die Digitalgrundsätze sollen europäische Werte im digitalen Raum wahren und bei der zukünftigen Formulierung von Regeln Orientierung bieten. Sie sollen auf Grundlage eines breit angelegten bis September 2021 laufenden Konsultationsprozesses erarbeitet werden, den die Kommission mit dem Ziel initiierte, eine feierliche interinstitutionelle Vereinbarung zwischen Europäischem Parlament, Rat und Kommission zu schließen. Im digitalen Kompass formulierte die Kommission Pläne in den vier Kernbereichen Fertigkeiten, Infrastruktur, Unternehmen und öffentliche Dienste. Mit konkreten Zielen für die Ausbildung

44 Europäische Kommission: Mitteilung der Kommission an das Europäische Parlament, den Rat, den Europäischen Wirtschafts- und Sozialausschuss und den Ausschuss der Regionen - Digitaler Kompass 2030: der europäische Weg in die digitale Dekade, 9. März 2021, COM(2021) 118 final, S. 2.

45 Europäischer Rat: Außerordentliche Tagung des Europäischen Rates (1. und 2. Oktober 2020) - Schlussfolgerungen, EUCO 13/20.

46 Europäischer Rat: Tagung des Europäischen Rates (15. und 16. Oktober 2020) - Schlussfolgerungen, EUCO $15 / 20$, S. 4.

47 Europäischer Rat: Tagung des Europäischen Rates (10. und 11. Dezember 2020) - Schlussfolgerungen, EUCO $22 / 20$.

48 Europäische Kommission: Speech by Executive Vice-President Vestager at the press conference on Europe's Digital Decade: 2030 Digital Targets, 9. März 2021, SPEECH/21/1092. 
digitaler Kompetenzen in der Gesellschaft, der Entwicklung von IT-Fachkräften, bessere Netzanbindung (u. a. 5G), Innovationen in den Halbleiter-, Cloud- und Quantentechnologien, den Anteil von Big Data und KI-nutzenden Unternehmen, eine neue Industriestrategie und die digitalisierte Verwaltung trug sie dabei Elemente für ein Reformprogramm zusammen, dessen Umsetzung bereits 2020 mit einer Reihe von Initiativen begonnen hatte. Um diese Ziele zu verwirklichen, entwickelte die Kommission den „Weg in die digitale Dekade“, der als Governance-Rahmen einen Überwachungs-, Berichts- und Planungsmechanismus sowie Kooperationsstrukturen mit und zwischen den Mitgliedstaaten, u. a. für digitale Mehrländer- und Großprojekte, vorsieht. ${ }^{49}$

Die Mitgliedstaaten führten die EU-Agenda im Laufe des Jahres 2021 in der Verbindung von grünem und digitalem Wandel weiter aus, wobei sie diese mit Europas Sicherheitsinteressen, auch in Hinblick auf aktuelle Cyberaktivitäten gegen einzelne Mitgliedstaaten sowie Wettbewerbsfähigkeit und wirtschaftliche Erholung in Verbindung setzten. Zu den Arbeitsaufträgen an die Kommission und die gesetzgebenden Organe gehörte es, die bereits angelaufenen Vorhaben voranzutreiben und zusätzliche kritische Technologien und strategische Sektoren der Zukunft zu bestimmen, die Europas digitale Souveränität stärken. ${ }^{50}$

\section{Digitalisierung in zentralen Handlungsfeldern}

Die Entwicklungen im vergangenen Jahrzehnt zeigen, wie umfangreich Digitalisierung heute durch EU-Politiken adressiert wird. Dabei haben sich umfassende Initiativen entlang zentraler Handlungsfelder entsponnen, die sich in fünf miteinander verbundene, teils überlappende Bereiche kategorisieren lassen:

(1) Digitale Infrastruktur und Cybersicherheit: Im Zentrum dieses Bereichs stehen die Themen Konnektivität (insbesondere Breitbandversorgung und 5G, auch für ländliche Gebiete) auf Grundlage physischer und Dienstleistungsinfrastrukturen (z. B. Cloud-Dienste, Hochleistungsrechnen oder das digitale Ökosystem Gaia-X) sowie Fragestellungen im Bereich der Daten- und IT-Sicherheit. Cybersicherheit ist aufgrund ihrer übergreifenden Natur für so gut wie alle Bereiche der Digitalisierung zentral. Darüber hinaus sind die Weltraumprogramme der EU, einschließlich des Erdbeobachtungsprogramms Copernicus und des Satelliten-Navigationssystems Galileo, aufgrund der fundamentalen Rolle von Satelliten für das Funktionieren vieler digitaler Anwendungen von infrastruktureller Bedeutung.

(2) Wirtschaft und digitaler Binnenmarkt: Hier adressiert die EU den Handel von Waren und Dienstleistungen im Internet (Online-Handel, digitale Produkte), den Aufbau eines gemeinsamen europäischen Datenraums mit diskriminierungsfreiem Internetzugang, die Regulierung von digitalen Diensten und Online-Plattformen, die Förderung kleiner, mittelständischer und Start-up-Unternehmen der Digitalwirtschaft und datenbasierter Geschäftsmodelle (Datenwirtschaft) sowie Maßnahmen zur Modernisierung der

49 Europäische Kommission: Vorschlag für einen Beschluss des Europäischen Parlaments und des Rates über das Politikprogramm für 2030 „Weg in die digitale Dekade“, 15. September 2021, COM(2021) 574 final.

50 Minister der Mitgliedstaaten der Europäischen Union: A Green and Digital Transformation of the EU. Ministerial Declaration, 19. März 2021, abrufbar unter: https://www.portugal.gov.pt/download-ficheiros/ficheiro.as px?v=\%3D\%3DBQAAAB\%2BLCAAAAAAABAAzNDQxMwMAT7AwdwUAAAA\%3D (letzter Zugriff: 19.10.2021); Europäischer Rat: Erklärung der Mitglieder des Europäischen Rates, 25. März 2021, SN 18/21. 
europäischen Industrie vor dem Hintergrund der Digitalisierung und für den Übergang zu einem Modell der Kreislaufwirtschaft. Die Besteuerung grenzüberschreitend agierender Digitalunternehmen und das digitale Finanzwesen gehören ebenfalls in den Bereich. Eine jüngere Entwicklung in diesem Zusammenhang ist die Konzeption des digitalen Euro.

(3) Forschung für digitale Technologie und Innovation: Eng verbunden mit den beiden vorgenannten Bereichen sind EU-Initiativen zu KI (einschließlich ethischer Fragestellungen) und anderen Schlüsseltechnologien, Halbleitern, Batterien und Blockchain. Neben Maßnahmen zum Hochleistungsrechnen fallen weitere Projekte aus dem Bereich Forschung perspektivisch in den Bereich Infrastruktur und Cybersicherheit, so z. B. „European Quantum Communication Infrastructure“ (EuroQCI) im Feld der Quantenkommunikation.

(4) Digitale Gesellschaft: In diesem Bereich lassen sich sowohl Regulierungen im Bereich Datenschutz (insbesondere die prominente DSGVO) als auch die Entwicklung von EU-weiten Digitalgrundsätzen und Initiativen - teilweise als freiwillige Verhaltenskodexe, teilweise als Aktionspläne - zum Schutz vor Desinformation oder der Verbreitung von illegaler Online-Hetze sowie zur Demokratie- und Medienförderung fassen. Damit verbunden sind Bemühungen um die digitale Kreativwirtschaft und die Aus- und Fortbildung digitaler Kompetenzen der BürgerInnen. Governance und Regierungsführung, insbesondere die Stärkung digitaler Verwaltung und öffentlicher Dienstleistungen, etwa unter Nutzung öffentlicher Daten oder einer europäischen digitalen Identität (eIDAS, eID), stellen einen wichtigen Teilbereich der digitalen Gesellschaft an der Schnittstelle zwischen BürgerInnen, Verwaltung und Politik dar. Auf die Verbesserung der grenzüberschreitenden Kommunikation und Datennutzung zielt die Digitalisierung der Justiz (e-CODEX). Nicht zuletzt durch digitale Lösungen im Kontext der COVID-19-Pandemie (z. B. das digitale Impfzertifikat) ist darüber hinaus auch der Gesundheitsbereich und die Entwicklung eines europäischen Gesundheitsdatenraums als Handlungsfeld auf EU-Ebene erkannt worden.

(5) Digitalisierung in den Außenbeziehungen: Hier geht es um die genannten Bereiche in ihrer jeweiligen Außendimension. Darunter fallen bspw. internationale Partnerschaften für die Setzung von Normen und Standards oder grenzüberschreitende Regulierungsfragen, aber auch interkontinentale Infrastruktur und die internationale Besteuerung der Digitalwirtschaft. Weitere Kernelemente sind die „Cyber Diplomacy Toolbox“, die diplomatische Reaktionen der Mitgliedstaaten auf Cyberangriffe regelt, und der EU-Politikrahmen für die Cyberabwehr. Cybersicherheit wird auch im Rahmen der Ständigen Strukturierten Zusammenarbeit (PESCO) adressiert. Einzelnen Ländern wie Russland wird über das Feld der Cybersicherheit hinaus besonderes Augenmerk geschenkt, nicht zuletzt mit Blick auf die Verbreitung von Desinformation. Darüber hinaus weisen neben Datenverkehr mit Drittländern in Handelsabkommen die Beziehungen im Rahmen der Nachbarschafts- und Erweiterungspolitik, insbesondere zum westlichen Balkan und die Beziehungen zu afrikanischen Staaten, nicht zuletzt innerhalb der Initiative „Digitalisation for Development“ (D4D) Bezüge zu Digitalisierungsprozessen auf. Dazu zählen Maßnahmen wie Wissenskooperation oder Multi-Stakeholder-Partnerschaften, um digitale Investitionen zu fördern und digitale Transformationsprozesse zu unterstützen. 


\section{Vier Lesarten der Digitalisierung}

Im Zusammenhang mit Digitalisierung wird auf EU-Ebene häufig der Begriff der Strategie genutzt. Dahinter steht die Absicht, den politischen Ansatz zur Steuerung der Digitalisierung, ihrer Verbindung mit und Auswirkungen auf Wirtschaft, Gesellschaft und Umwelt zu fassen. Beispiele sind die Strategie für einen digitalen Binnenmarkt und die sogenannte Digitalstrategie zur Gestaltung der digitalen Zukunft Europas, die Strategie für Cybersicherheit, die Industriestrategie ${ }^{51}$, die Datenstrategie, die Strategie für kleine und mittlere Unternehmen (KMU) für ein nachhaltiges und digitales Europa ${ }^{52}$ oder die EU-Strategie für eine Sicherheitsunion ${ }^{53}$. Die Strategien zielen dabei - vielmals miteinander verknüpft und auf Interdependenzen hinweisend - auf verschiedene Handlungsfelder. Darüber hinaus gibt es Rahmenwerke, etwa den Green Deal oder den MFR, die die europäische Ausgestaltung der Digitalisierung mitbestimmen. Vor diesem Hintergrund stellt sich die Frage, welches grundlegende Verständnis von Digitalisierung sich in der EU herausgebildet hat und welche Kernelemente einer über Einzelstrategien hinausgehenden, übergreifenden Digitalisierungsstrategie erkennbar werden.

Neben der Situationsanalyse (wie ist die Ausgangslage für die EU?) und der Zielformulierung (was soll die EU erreichen?) gehört das Zielimage (wie soll die EU wahrgenommen werden und welche Imagefaktoren werden betont?) zu den Kernelementen politischer Strategien. Hierbei spielt auch das Framing von Digitalisierung und der Rolle der EU in den politischen Schlüsselwerken eine Rolle, das durch Benennung, Selektierung und Kategorisierung von Informationen Bedeutungsbestimmungen vornimmt. Auf diese Weise wird eine grundlegende Erzählung, ein Narrativ der Digitalisierung und der Rolle der EU gestaltet, das mit Situationsanalyse und Zielformulierungen in Verbindung steht. Entlang dieser Elemente lassen sich vier Deutungsrahmen bzw. Lesarten unterscheiden, die zentrale Bausteine einer übergreifenden EU-Digitalisierungsstrategie liefern.

\section{Das geopolitische Framing: digitale Souveränität}

Digitale Souveränität ist eine Lesart von Digitalisierung und der Rolle der EU, die in der politischen (und politikwissenschaftlichen) Debatte derzeit besonders prominent in Erscheinung tritt. ${ }^{54}$ Die deutsche EU-Ratspräsidentschaft 2020 nutzte den Begriff als Leitmotiv europäischer Digitalpolitik. ${ }^{55}$ Hierbei ist digitale Souveränität in die Diskussion

51 Europäische Kommission: Mitteilung der Kommission an das Europäische Parlament, den Rat, den Europäischen Wirtschafts- und Sozialausschuss und den Ausschuss der Regionen. Eine neue Industriestrategie für Europa, 10. März 2020, COM(2020) 102 final.

52 Europäische Kommission: Mitteilung der Kommission an das Europäische Parlament, den Rat, den Europäischen Wirtschafts- und Sozialausschuss und den Ausschuss der Regionen. Eine KMU-Strategie für ein nachhaltiges und digitales Europa, 10. März 2020, $\operatorname{COM}(2020) 103$ final.

53 Europäische Kommission: Mitteilung der Kommission an das Europäische Parlament, den Rat, den Europäischen Wirtschafts- und Sozialausschuss und den Ausschuss der Regionen - EU-Strategie für eine Sicherheitsunion, 24. Juli 2020, $\operatorname{COM}(2020) 605$ final.

54 Vgl. u. a. Europäische Kommission: Präsidentin von der Leyens Rede zur Lage der Union bei der Plenartagung des Europäischen Parlaments, 16. September 2020, SPEECH/20/1655; Guntram Wolff/Niclas Poitiers/Pauline Weil: Sovereignty and digital interdependence, in: Daniel Fiott (Hrsg.): European Sovereignty. Strategy and interdependence, Luxemburg 2021, S. 16-22; Anke S. Obendiek: Take back control? Digital sovereignty and a vision for Europe, Hertie School Jacques Delors Centre: Policy Paper, 12. Mai 2021.

55 Deutsche Präsidentschaft im Rat der EU: Ausbau der digitalen Souveränität, 2020, abrufbar unter: https://www .eu2020.de/eu2020-en/eu-digitalisation-technology-sovereignty/2352828 (letzter Zugriff: 30.9.2021); Gabrielle 
um strategische Autonomie der EU eingebettet, die bereits in der Globalen Strategie für die Außen- und Sicherheitspolitik der EU Bezüge zu Politikfeldern mit erheblichen Digitalbezügen (Cybersicherheit, Terrorismusbekämpfung, strategische Kommunikation) aufwies. ${ }^{56}$ Kern des Konzepts ist die Frage nach der Fähigkeit der EU, für den digitalen Wandel selbstbestimmte Entscheidungen treffen zu können - frei von ausländischen Zwängen und möglichen zukünftigen Abhängigkeiten. ${ }^{57}$ Dem tiefgreifenden Transformationscharakter der Digitalisierung entsprechend werden in diesem Zusammenhang nicht nur die Kontrolle des Cyberraums mit seinen technischen digitalen Systemen (Hardware, Software, Infrastruktur, Standards und Protokolle), Cybersicherheit sowie Datensammlung, -fluss und -zugriff angesprochen. Die Frage nach digitaler Souveränität kann auch in wirtschaftliche und gesellschaftliche Bereiche hineinreichen, etwa wenn Digitalisierung Wettbewerbsbedingungen verändert oder demokratische Prozesse beeinflusst. ${ }^{58}$

Das Framing der digitalen Souveränität betont, dass die EU in technologischen Rückstand und damit in Abhängigkeiten gegenüber Drittstaaten und dort ansässigen Unternehmen geraten sei. Dadurch gewinnt auch die sicherheitspolitische Dimension der Digitalisierung an Bedeutung. Diese Verbindung zeigt sich besonders in den Feldern Cloud-Infrastruktur, Cybersicherheit und Aufbau des 5G-Mobilfunkstandards. 5G ist Grundlage technologischer Innovationen in vielen Bereichen, z. B. für die automatisierte Steuerung in Transport und Mobilität. Es wird als vital u. a. für die kritische öffentliche Infrastruktur der Zukunft betrachtet. Die Verwendung von Bauteilen chinesischer Hersteller wird deshalb als mögliches Risiko für die nationale Sicherheit diskutiert. Bei Cloud-Diensten bestehen Abhängigkeiten etwa mit Bezug zur Versorgungssicherheit oder unrechtmäßigem Datenzugriff durch Drittländer. Mit dem Konzept der digitalen Souveränität spricht die EU darüber hinaus Abhängigkeiten von Importen an, insbesondere von kritischen Rohstoffen oder wichtigen Bauteilen wie Halbleitern. ${ }^{59}$

Die größten Abhängigkeiten bestehen gegenüber China, den USA und einer Reihe (süd-)ostasiatischer Staaten. Das auf Marktfreiheit und global operierenden Tech-Giganten (Google, Amazon, Meta, Microsoft, Apple) aufbauende US-amerikanische kommerzielle, (überwachungs-)kapitalistische Modell auf der einen Seite ${ }^{60}$ und das chinesische autoritäre staatliche Überwachungsmodell auf der anderen Seite sind Gravitationszentren für über-

Heyvaert: Europe's Digital decade: Game on, Centre International de Formation Européenne: CIFE Policy Paper 2/2021, S. 1-3.

56 Rat der Europäischen Union: Eine Globale Strategie für die Außen- und Sicherheitspolitik der Europäischen Union, 28. Juni 2016, 10715/16, S. 7. Zum Konzept der strategischen Autonomie vgl. u. a. Niklas Helwig (Hrsg.): Strategic autonomy and the transformation of the EU. New agendas for security, diplomacy, trade and technology, Helsinki 2021.

57 Europäische Kommission: Shaping Europe's digital future: op-ed by Ursula von der Leyen, President of the European Commission, 19. Februar 2020, AC/20/260, S. 12; Steiner/Grzymek: Digital Sovereignty in the EU, 2020, S. 7.

58 Lokke Moerel/Paul Timmers: Reflections on Digital Sovereignty, in: Research in Focus 1/2021, S. 1-33, hier S. 4; Luciano Floridi: The fight for digital sovereignty: what it is, and why it matters, especially for the EU, in: Philosophy and Technology 3/2020, S. 369-378, hier S. 370-371.

59 Europäische Kommission: Mitteilung der Kommission an das Europäische Parlament, den Rat, den Europäischen Wirtschafts- und Sozialausschuss und den Ausschuss der Regionen. Widerstandsfähigkeit der EU bei kritischen Rohstoffen: Einen Pfad hin zu größerer Sicherheit und Nachhaltigkeit abstecken, 3. September 2020, $\operatorname{COM}(2020) 474$ final.

60 Shoshana Zuboff: The Age of Surveillance Capitalism: The Fight for a Human Future at the New Frontier of Power, New York 2019. 
greifende Digitalisierungskonzepte weltweit. Die EU beobachtet deshalb Spannungen zwischen den USA und China mit großer Aufmerksamkeit, da Technologie hier Gegenstand geopolitischer Auseinandersetzungen wird.

Auf dieser Situationsanalyse aufbauend formuliert die EU als zentrale Ziele insbesondere die Stärkung der eigenen industriellen und technologischen Basis - mit Blick auf das Erzielen von Größenvorteilen - und die Förderung von Tech-Unternehmen sowie Forschung und Entwicklung digitaler Technologien. ${ }^{61}$ Die neue Industriestrategie adressiert entsprechend strategische Abhängigkeiten und Vulnerabilitäten in Lieferketten und Cyberbedrohungen. Der Anspruch, eigene Regeln festlegen zu können und über strategische digitale Kapazitäten zu verfügen, reicht auch in die Außenbeziehungen hinein, die z. B. Beiträge zur Etablierung globaler Standards und Normen leisten oder die Entwicklung interkontinentaler Datengateways von geopolitischer Relevanz fördern sollen. ${ }^{62}$

Von dieser Problemanalyse und Zielformulierung ausgehend skizziert die EU ein Selbstbild des "dritten Wegs" der Digitalisierung, der sich nicht nur vom Systemrivalen China, sondern auch gegenüber den USA absetzt - insbesondere in punkto Datenschutz, freiem Daten- und Informationsfluss sowie Achtung der Privatsphäre. Über technologische Innovationskraft in Verbindung mit der DSGVO und den Initiativen zu digitalen Rechten und KI versucht sich die EU als souveräne Regulierungsmacht und wertebasiertes, auf den Menschen ausgerichtetes alternatives Referenzmodell zu präsentieren und durchzusetzen. ${ }^{63}$ Dafür bedarf es in Zeiten globaler Machtverschiebungen nicht nur digitaler Handlungsfähigkeit, sondern Unabhängigkeit und - angesichts des technologischen Rückstands - entsprechender Investitionen.

\section{Das umweltpolitische Framing: grüne Transformation}

Das umweltpolitische Framing nahm gemeinsam mit der Veröffentlichung des Green Deal Fahrt auf. Die Situationsanalyse dieser Lesart fußt auf der Einsicht, dass Erhalt natürlicher Ökosysteme, nachhaltige Nutzung der Ressourcen und Verbesserung der Gesundheit Prioritäten zukunftsgerechter Politik sein müssen. Digitalisierung spielt in diesem Zusammenhang eine zentrale Rolle, da sie als Bedingung für den notwendigen Wandel in den genannten Bereichen angesehen wird. Dementsprechend heißt es im Green Deal: „Digitale Technologien sind eine entscheidende Voraussetzung für die Verwirklichung der Nachhaltigkeitsziele des Grünen Deals in vielen verschiedenen Sektoren. "64 Digitalisierung wird insbesondere mit den Klimazielen der EU, dem europäischen Energiemarkt und dem Umbau von Wirtschaft und Industrie in Verbindung gestellt, in der Erwartung, dass digitale Technik intensivere und effizientere Ressourcennutzung erlaubt. Zugleich betonen die Mitgliedstaaten die Relevanz des ökologischen Wandels des digitalen Sektors zugunsten

61 Europäischer Rat: Außerordentliche Tagung des Europäischen Rates (1. und 2. Oktober) - Schlussfolgerungen, EUCO 13/20, S. 3-4.

62 Ministers from the Member States of the European Union: European Data Gateways as a key element of the EU's Digital Decade. Ministerial Declaration, 19. März 2021, abrufbar unter: https://www.portugal.gov.pt/dow nloadficheiros/ficheiro.aspx?v=\%3D\%3DBQAAAB\%2BLCAAAAAAABAAzNDQxMwUA9eE57gUAAAA\%3 D (letzter Zugriff: 19.10.2021).

63 Ingrid Schneider: Datenschutz, KI und digitale Rechte in Mexiko - Europa als Referenzmodell?, in: Zeitschrift für Bürgerrechte und Gesellschaftspolitik 3-4/2020, S. 131-145; Anu Bradford: The Brussels Effect. How the European Union Rules the World, New York 2020.

64 Europäische Kommission: Der europäische Grüne Deal, Dezember 2019, S. 11. 
einer nachhaltigkeitsorientierten Ausrichtung, nicht zuletzt mit Blick auf die Auswirkungen von Rechenzentren und Cloud-Infrastrukturen auf den Energieverbrauch.

Ökologischer und digitaler Wandel werden somit als zweifache oder doppelte Transformation („twin transitions“) und als voneinander untrennbar dargestellt. Die Lesart schlägt sich in zahlreichen aktuellen Zielformulierungen der EU nieder. Bspw. soll Europa als Leitmarkt für saubere Technologie etabliert und der Wandel zugunsten einer digital gestützten nachhaltigen Lebensmittelerzeugung und europäischen Kreislaufwirtschaft angeschoben werden. ${ }^{65}$ Die "grüne und digitale Transformation der EU“ adressiert auch die Mobilitätsund Bausektoren sowie die Nutzung von KI, um nachhaltige Lösungen zu finden. ${ }^{66}$ Unter dem Leitmotiv der „Digitalisierung zum Wohle der Umwelt“ unterstützt die EU als Schlüsselbranche für den Kampf gegen Klimawandel sowohl Allianzen von IKT-Unternehmen, die grünen und digitalen Wandel verbinden, als auch den „Aufbau eines Datenraums für den europäischen Grünen Deal, in dem öffentliche und private Akteure einbezogen sind. “67 Die Ziele finden auch Niederschlag in der finanziellen Ausstattung des MFR und NGEU und reichen darüber hinaus in den Bereich der auswärtigen Beziehungen, etwa wenn die Cybersicherheitsstrategie den „Aufbau externer Cyberkapazitäten [...] im Einklang mit [...] der Agenda 2030 für nachhaltige Entwicklung "68 oder die Partnerschaft mit Afrika Zusammenarbeit in digitaler und wissensbasierter Wirtschaft vorsieht. ${ }^{69}$ In der Zusammenarbeit mit den Vereinten Nationen fordert die EU die Entwicklung einer weltweiten Umweltdatenstrategie. Grüne digitale Diplomatie wird auch als Chance für die europäische Außen- und Sicherheitspolitik diskutiert. ${ }^{70}$

Durch die Betonung des Digitalen im Green Deal und des Grünen in der digitalen Agenda baut insbesondere die Kommission drei Imagefaktoren auf: Erstens wird die grüne Transformation über die namentliche Anlehnung an den „New Deal“ der USA in den 1930er Jahren in die Tradition historischer Wirtschaftsreformen gestellt. Zweitens bildet das Selbstbild des digitalen grünen Deals einen Kontrast zu den umweltpolitisch fragwürdigen Deals des ehemaligen US-Präsidenten Donald Trump. Drittens verleiht das Konzept der nachhaltigen Digitalisierung der EU einen grünen und modernen Anstrich, der die erheblichen Beiträge des Kontinents zum globalen Ausstoß von Klimagasen mit

65 Europäische Kommission: Eine neue Industriestrategie für Europa, März 2020; Europäische Kommission: Mitteilung der Kommission an das Europäische Parlament, den Rat, den Europäischen Wirtschafts- und Sozialausschuss und den Ausschuss der Regionen. Ein neuer Aktionsplan für die Kreislaufwirtschaft. Für ein saubereres und wettbewerbsfähigeres Europa, 11. März 2020, COM(2020) 98 final; Europäische Kommission: Mitteilung der Kommission an das Europäische Parlament, den Rat, den Europäischen Wirtschafts- und Sozialausschuss und den Ausschuss der Regionen: „Vom Hof auf den Tisch“ - eine Strategie für ein faires, gesundes und umweltfreundliches Lebensmittelsystem, 20. Mai 2020, COM(2020) 381 final.

66 Minister der Mitgliedstaaten der Europäischen Union: A Green and Digital Transformation of the EU, März 2020.

67 Rat der Europäischen Union: Entwurf von Schlussfolgerungen des Rates zum Thema Digitalisierung zum Wohle der Umwelt - Billigung, 11. Dezember 2020, 13957/20.

68 Europäische Kommission/Sicherheitspolitik: Die Cybersicherheitsstrategie der EU für die digitale Dekade, Dezember 2020, S. 27.

69 Europäischer Rat: Tagung des Europäischen Rates (15. und 16. Oktober 2020) - Schlussfolgerungen, Oktober 2020, S. 4. Zur digitalen Partnerschaft zwischen Afrika und Europa vgl. auch Chux Daniels/Benedikt Erforth/Rob Floyd/Chloe Teevan: Strengthening the Digital Partnerships between Africa and Europe, Oktober 2020, abrufbar unter: https://acetforafrica.org/acet/wp-content/uploads/publications/2020/10/ETTG-Publicati on-Strengthening-the-digital-partnership-between-Africa-and-Europe.pdf (letzter Zugriff: 19.10.2021).

70 Patryk Pawlak/Fabio Barbero: Green Digital Diplomacy. Time for the EU to lead, European Union Institute for Security Studies: Brief 18/2021. 
einer positiven Transformationsagenda verbindet - und auf diese Weise versucht, sie auch rhetorisch einzufangen.

\section{Das gesellschaftspolitische Framing: Grundrechte und Schutz vor Bedrohungen}

Mit Blick auf gesellschaftspolitische Prozesse kann in den untersuchten Leitlinien ein Framing der Digitalisierung und der Rolle der EU identifiziert werden, das digitale Grundrechte, Schutz vor digitalen Bedrohungen und Vertrauen in digitale Dienste betont. Ausgangspunkt dieser Lesart ist, dass digitale Entwicklungen zahlreiche neue Gefahren für Staat, Gesellschaft und Wirtschaft mit sich bringen. Diese reichen von Cyberbedrohungen (Hacking, Erpressungssoftware, Identitätsdiebstahl) über Einschränkungen des Rechts auf Achtung des Privatlebens bis zu Herausforderungen bezüglich der „Meinungsfreiheit mit Zugang zu pluralistischen, verlässlichen und transparenten Informationen“71. Den Kern der Situationsanalyse macht aus, dass nicht alle digitalen Innovationen automatisch europäisches Recht in den digitalen Raum übertragen oder dort respektieren, wodurch nicht zuletzt demokratische Prozesse bedroht werden können. Gleichzeitig ist das Vertrauen der Bevölkerung in digitale Lösungen Voraussetzung für eine erfolgreiche Gestaltung und Nutzung von Digitalisierung in vielen Bereichen. Der Europäische Rat forderte entsprechend die Einhaltung und Durchsetzung nationaler und europäische Rechtsvorschriften zum Schutz der Privatsphäre und personenbezogener Daten europäischer BürgerInnen. ${ }^{72}$ Dazu gehören u. a. die Verletzung von Urheberrechten, Risiken, die von der Reichweite großer Online-Plattformen (z. B. soziale Medien) ausgehen, der Verkauf illegaler Güter oder die Verbreitung illegaler Online-Inhalte oder neue Mittel der Desinformation. Darüber hinaus erhebt die EU jedoch auch Anspruch auf die Gestaltung technologischer Fortschritte im Einklang mit europäischen Werten und Normen, etwa bei der Weiterentwicklung und Nutzung von Gesichtserkennungssoftware.

Daraus ergibt sich für die EU entlang der Leitbilder der „Technologie im Dienste der Menschen“ und der „offenen, demokratischen und nachhaltigen Gesellschaft" umfassender Handlungsbedarf, insbesondere um Datensicherheit, Datenschutz und Privatsphäre zu gewährleisten. Sie kann sich dabei den Rückenwind zu Nutzen machen, den die erfolgreiche, weltweit beachtete Einführung von Regeln zur Verarbeitung personenbezogener Daten im Rahmen der DSGVO erzeugt hat. Die Entwicklung einer sicheren, vertrauenswürdigen und ethisch vertretbaren künstlichen Intelligenz, ${ }^{73}$ die Regulierung großer Plattformen, ${ }^{74}$ Maßnahmen gegen Versuche, den Informationsraum zu manipulieren ${ }^{75}$ die Entwicklung

71 Europäische Kommission: Europas digitale Dekade: digitale Ziele für 2030, abrufbar unter: https://ec.europa .eu/info/strategy/priorities-2019-2024/europe-fit-digital-age/europes-digital-decade-digital-targets-2030_de (letzter Zugriff: 1.11.2021).

72 Europäischer Rat: Tagung des Europäischen Rates (22. März 2018) - Schlussfolgerungen, EUCO 1/18, S. 3.

73 Europäische Kommission: Vorschlag für eine Verordnung des Europäischen Parlaments und des Rates zur Festlegung harmonisierter Vorschriften für Künstliche Intelligenz (Gesetz über Künstliche Intelligenz) und zur Änderung bestimmter Rechtsakte der Union, 21. April 2021, COM(2021) 206 final.

74 Europäische Kommission: Vorschlag für eine Verordnung des Europäischen Parlaments und des Rates über einen Binnenmarkt für digitale Dienste (Gesetz über digitale Dienste) und zur Änderung der Richtlinie 2000/31/EG, 15. Dezember 2020, COM(2020) 825 final.

75 Europäische Kommission: EU Code of Practice on Disinformation, 1. Oktober 2021, abrufbar unter: https://di gital-strategy.ec.europa.eu/en/policies/code-practice-disinformation (letzter Zugriff: 19.10.2021); Europäische Kommission/Hohe Vertreterin der Europäischen Union für Außen- und Sicherheitspolitik: Aktionsplan gegen Desinformation, Dezember 2018. 
vertrauenswürdiger digitaler Identitäten ${ }^{76}$ und Aktionspläne für Medien und Demokratie ${ }^{77}$ stehen für diese Form der Digitalisierungsgestaltung durch die EU. Als zentraler Bestandteil der Gesamtvision vom digitalen Wandel in Europa wird die Verbesserung von digitaler Bildung und digitaler Kompetenzen benannt, so dass u. a. „die Fähigkeit, sich konstruktiv und sachkundig im digitalen Umfeld zu bewegen“ dazu beitragen kann, „Resilienz in Zeiten des rasanten technologischen Wandels und der Globalisierung zu stärken." ${ }^{\text {"78 }}$ Mit der Befähigung zur „digitalen Autonomie des Einzelnen“ klingt auch die soziale Abfederung und Weiterbildung für Menschen mit, deren Jobs von Automatisierung bedroht sind.

Mit diesem Framing konstruiert die EU ein Zielimage des geschützten und zugleich offenen digitalen Raums, in den die Bevölkerung Vertrauen hat, in dem europäische Werte hochgehalten, Demokratie geschützt, Verträge gehütet und BürgerInnen vor neuen Bedrohungen bewahrt und mit mehr Rechten ausgestattet werden. Die Verteidigung dieses Raums hat auch eine außen- und sicherheitspolitische Komponente, etwa wenn ausländische Mächte im Rahmen der Desinformation oder des Diebstahls geistigen Eigentums angesprochen und die „Cyber Diplomacy Toolbox" als koordinierte Reaktion auf hybride Bedrohungen angeführt werden.

\section{Das wirtschaftspolitische Framing: Wachstum und Wettbewerbsfähigkeit}

Eine vierte Lesart hebt wirtschaftspolitische Aspekte wie Wachstum und Wettbewerbsfähigkeit hervor. Das Framing setzt bei zwei Ausgangspunkten an: Zum einen baut sie auf dem enormen Potenzial für Innovation, Wachstum und Beschäftigung durch Digitalisierung auf. In Anerkennung der Schlüsselrolle digitaler Technik für die Neugestaltung Europas versprechen Maßnahmen für elektronischen Geschäftsverkehr, bessere Rahmenbedingungen zur Förderung neuer Geschäftsmöglichkeiten, digitale Infrastruktur und Investitionen neben wirtschaftlicher Erneuerung auch erhebliche Zusatzeinnahmen. ${ }^{79}$ Zum anderen geht dieses Deutungsmuster von Gefahren für die Expansionschancen des europäischen Marktes aus, da Europa im globalen Vergleich an wirtschaftlicher Innovationsund Wettbewerbsfähigkeit verlieren und nicht stark genug in strategische internationale digitale Wertschöpfungsketten eingebunden sein könnte. So findet bspw. die mit CloudComputing verbundene Wertschöpfung größtenteils außerhalb der EU statt. Zentrales Instrument zur Realisierung sowohl des Innovations- und Wachstumspotenzials als auch der Steigerung der Wettbewerbsfähigkeit ist die Entwicklung des Binnenmarkts, dessen Freiheiten es umfänglich umzusetzen gilt.

76 Europäische Kommission: Vorschlag für eine Verordnung des Europäischen Parlaments und des Rates zur Änderung der Verordnung (EU) Nr. 910/2014 im Hinblick auf die Schaffung eines Rahmens für eine europäische digitale Identität, 3. Juni 2021, COM(2021) 281 final.

77 Europäische Kommission: Mitteilung der Kommission an das Europäische Parlament, den Rat, den Europäischen Wirtschafts- und Sozialausschuss und den Ausschuss der Regionen. Europas Medien in der digitalen Dekade: Ein Aktionsplan zur Unterstützung der Erholung und des Wandels, 3. Dezember 2020, COM(2020) 784 final; Europäische Kommission: Mitteilung der Kommission an das Europäische Parlament, den Rat, den Europäischen Wirtschafts- und Sozialausschuss und den Ausschuss der Regionen. Europäischer Aktionsplan für Demokratie, 3. Dezember 2020, COM(2020) 790 final.

78 Europäische Kommission: Mitteilung der Kommission an das Europäische Parlament, den Rat, den Europäischen Wirtschafts- und Sozialausschuss und den Ausschuss der Regionen zum Aktionsplan für digitale Bildung, 17. Januar 2018, COM(2018) 22 final, S. 3 und 5.

79 J. Scott Marcus/Georgios Petropoulos/Timothy Yeung: Contribution to Growth: The European Digital Single Market - Delivering economic benefits for citizens and businesses. Study requested by the IMCO Committee of the European Parliament, Brüssel 2019. 
Vor diesem Hintergrund benennt die Strategie für einen digitalen Binnenmarkt die Schaffung eines günstigen Investitionsklimas für digitale Netze, die Forschung und innovative Unternehmen als Hauptziel. Unter dem Leitmotiv der Vollendung des digitalen Binnenmarkts werden die Entwicklung von digitaler Infrastruktur und Kompetenzen, die Förderung von E-Commerce und digitalen Dienstleistungen als zentrale Instrumente gesehen, es Unternehmen und BürgerInnen zu ermöglichen, von den Vorteilen der Digitalisierung zu profitieren. Bedenken in Bezug auf „Sicherheit, die Wahrung der Grundrechte und den Datenschutz im Allgemeinen ${ }^{\text {"80 }}$ werden in dieser Perspektive als Schlüsselfaktoren für das Vertrauen von europäischen Unternehmen und BürgerInnen in digitale Lösungen gesehen.

Bei diesem wirtschaftspolitischen Framing geht es also um die konkreten Vorzüge eines integrierten Binnenmarkts für europäische Unternehmen und gesellschaftliche Entwicklung. Die Befreiung von den zusätzlichen Kosten des Roamings für Telefonie oder Datennutzung bei grenzüberschreitenden Reisen in Europa gehört zu den prominenten Initiativen in diesem Zusammenhang. Förderung von Forschung, KMU und Start-ups, ein digitalisierter öffentlicher Sektor und der Zugang zu Rohstoffen und funktionierenden Lieferketten werden somit auch mehr als Motor für die ökonomische Erneuerung und Weiterentwicklung Europas dargestellt und weniger als Faktoren in einem globalen Systemwettbewerb. Zugleich verweist auch dieses Verständnis aufgrund der wirtschaftlichen Verflechtungen auf globale Wettbewerbsfähigkeit. Ziel ist es nicht nur, die Position europäischer Unternehmen gegenüber ausländischen Mitbewerbern global zu stärken, nicht zuletzt mit Blick auf Besteuerung, sondern auch den Rahmen für fairen Wettbewerb herzustellen. Wenngleich die Kernelemente dieser Lesart besonders mit der digitalen Binnenmarkstrategie von 2015 verankert wurden, wird der digitale Wandel auch als „wesentliche Komponente der Reaktion der EU auf die durch die COVID-19-Pandemie ausgelöste Wirtschaftskrise“ ${ }^{\text {"81 }}$ erkannt.

Im Kern entwirft die EU durch dieses Framing ein Zielimage von Prosperität, ökonomischer Kraft und Wettbewerbsfähigkeit. Damit baut sie auf den grundlegendsten Versprechungen des europäischen Integrationsprojekts auf. Die wirtschaftliche Stärke als Integrationsgemeinschaft geht dabei mit der Digitalisierung des gemeinsamen Marktes einher.

\section{Kernelemente und Zukunft der europäischen Digitalisierungsstrategie}

Digitalisierung und Digitalpolitik haben sich in den vergangenen Jahren in der EU rasant entwickelt. Spätestens seit 2015 hat sich ein Spektrum an Aktivitäten entfaltet, das der bereichsübergreifenden Eigenschaft der Digitalisierung entsprechend breit ist. Die Entwicklung erfolgte in drei ineinandergreifenden Phasen, die für die Digitalagenda jeweils neue Prioritäten mit sich brachten. Die Entwicklung ging mit dem Aufbau und der Stärkung institutioneller Strukturen einher, wie bspw. das Ressort „Ein Europa für das digitale Zeitalter" von Kommissions-Vizepräsidentin Vestager und die Cybersicherheitsagentur

80 Europäische Kommission: Mitteilung der Kommission an das Europäische Parlament, den Rat, den Europäischen Wirtschafts- und Sozialausschuss und den Ausschuss der Regionen - Strategie für einen Digitalen Binnenmarkt für Europa, 6. Mai 2015, COM(2015) 192 final, S. 17.

81 Rat der Europäischen Union: Gestaltung der digitalen Zukunft Europas - Schlussfolgerungen des Rates (9. Juni 2020), S. 3. 
ENISA zeigen. Zugleich weisen die Entwicklungsphasen thematische Gemeinsamkeiten und Kontinuitäten auf, bspw. bezüglich der Förderung digitaler Infrastruktur oder wirtschaftlicher Innovationen. Eine Vielzahl der Initiativen, die durch politische Leitlinien und Strategiepapiere angestoßen wurden, stammt aus den vergangenen zwei Jahren. Einige von ihnen zählen zu den aktuell „wichtigsten politischen Strategien, insbesondere [die Strategien] des europäischen Grünen Deals, der digitalen Revolution und der Resilienz ${ }^{\text {“82. }}$.

Vor dem Hintergrund der dynamischen thematischen Erweiterung und inhaltlichen Vertiefung, den Institutionalisierungsschritten und erheblichen Ressourcenzuweisungen ist dieser Beitrag dem Verständnis von Digitalisierung und der Rolle der EU bei der Gestaltung von Digitalisierung auf europäischer Ebene nachgegangen. Strukturierung der Entwicklung und der heutigen Handlungsfelder lassen Digitalisierungsverständnis und Kernelemente einer politikfeldübergreifenden Digitalisierungsstrategie erkennen. Hier kann die Analyse vier Deutungsrahmen von Digitalisierung unterscheiden, in die die EU ihre Handlungen einbettet. Zeitpunkt der Einführung und Schwerpunktsetzung unterscheiden die geopolitischen (verstärkt seit 2017), umweltpolitischen (verstärkt seit 2019), gesellschaftspolitischen und wirtschaftspolitischen (beide verstärkt nach 2015 und erneut 2020) Framings. Sie schließen sich jedoch nicht aus. Vielmehr bedient sie die EU parallel und führt sie in ihrem aktuellen Ansatz zusammen.

Die Kernelemente einer politikfeldübergreifenden EU-Digitalisierungsstrategie lassen sich daher nicht als ein scharf abgestecktes, einheitliches Profil formulieren. Es zeigt sich allerdings eine Konvergenz aus diversen Teilstrategien zugunsten eines grundsätzlichen Verständnisses dessen, was Digitalisierung als Herausforderung und Chance ausmacht, und was die angestrebte Rolle der EU dabei ist: Digitalisierung nach eigenen Regeln und Werten auf Grundlage technologischer Souveränität als Schlüssel zu klima- und umweltgerechter, nachhaltiger Entwicklung einer wettbewerbsfähigen europäischen Wirtschaftszone und einer offenen, demokratischen Gesellschaft. ${ }^{83}$

Digitalisierung wird die EU in Zukunft noch mehr beschäftigen, wenn sie diese zielgerichteter und im Nutzen aller vorantreiben will. Zwischen den Mitgliedstaaten, zwischen ländlichem und städtischem Raum und zwischen Alters- und Bildungsschichten besteht eine „digitale Kluft“. Die Herausforderungen, die aus der Digitalisierung erwachsen, werden zunehmen, nicht nur im Zuge „digitaler Hyperkonnektivität“, sondern auch durch Druck mit digitalen Instrumenten von innen und außen auf das demokratische Modell der Union. ${ }^{84}$ Freiheitsrechte im Internet werden derzeit global immer stärker bedroht, freie Meinungsäußerung weiter eingeschränkt und der Ruf nach Regulierung großer Tech-Giganten lauter. ${ }^{85}$ Ein gemeinsames Verständnis von Digitalisierung und damit verbundene „kollektive Prinzipien, regulatorische Rahmenbedingungen und ethisch-begründete Gren-

82 Europäischer Rat: Außerordentliche Tagung des Europäischen Rates (17., 18., 19., 20. und 21. Juli 2020) Schlussfolgerungen, EUCO 10/20, S. 2.

83 Europäische Kommission: Rede der Präsidentin von der Leyen zur Lage der Union - 2021. Die Seele unserer Union stärken, Straßburg, 15. September 2021, SPEECH/21/4701.

84 Europäische Kommission: Mitteilung der Kommission an das Europäische Parlament, den Rat, den Europäischen Wirtschafts- und Sozialausschuss und den Ausschuss der Regionen. Strategische Vorausschau $2021-$ Die Handlungsfähigkeit und Handlungsfreiheit der EU, 8. September 2021, COM(2021) 750 final, S. 3-4.

85 Funk Shahbaz/Allie Funk: Freedom on the Net 2021. The Global Drive to Control Big Tech, Washington 2021. 


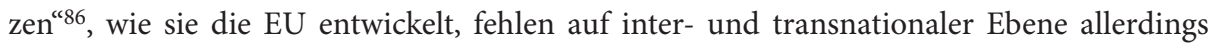
noch vollständig und ihre Entwicklung kann auch nicht auf ein funktionierendes System globaler Governance aufbauen. Dennoch sind erfolgreiche inklusive Verständigungsprozesse darüber, wie eine gemeinsame digitale Zukunft aussehen soll - mit staatlichen und nichtstaatlichen Akteuren aus allen Erdteilen - letztlich Schlüssel für die Förderung europäischer Ideen und Vorstellungen.

Für die EU und ihre Mitgliedstaaten geht es also um nichts Geringeres, inmitten globaler Machtverschiebungen in einem komplexen Feld voll Interdependenzen interund transnational koordiniert die Voraussetzungen dafür zu schaffen, den Megatrend Digitalisierung zugunsten einer nachhaltigen Entwicklung zu steuern. Diese Entwicklung muss die umwelt- und klimapolitischen Gebote unserer Zeit - insbesondere im Dreieck Digitalisierung/Energie/ $\mathrm{CO}_{2}$-Emissionen - berücksichtigen und sowohl den Ansprüchen des europäischen Werte- und Rechtssystems als auch der europäischen Öffentlichkeit ${ }^{87}$ genügen.

86 Wissenschaftlicher Beirat der Bundesregierung Globale Umweltveränderungen: Unsere gemeinsame digitale Zukunft, 2019, S. 6.

87 Vgl. u. a. Europäische Kommission: Flash Eurobarometer 443 - ePrivacy, 2016, abrufbar unter: https://data.eur opa.eu/data/datasets/s2124_443_eng?locale=en (letzter Zugriff: 19.10.2021). 